\title{
Gradient Yamabe Solitons on Multiply Warped Product Manifolds
}

\author{
Fatma Karaca* \\ (Communicated by Uday Chand De)
}

\begin{abstract}
We consider gradient Yamabe solitons on multiply warped product manifolds. We find the necessary and sufficient conditions for multiply warped product manifolds to be gradient Yamabe solitons.
\end{abstract}

Keywords: Yamabe soliton; gradient Yamabe soliton; multiply warped product.

AMS Subject Classification (2010): Primary: 53C25 ; Secondary: 53C21; $58 E 11$.

\section{Introduction}

Let $(M, g)$ be a semi-Riemannian manifold. If there exists a smooth vector field $X \in \chi(M)$ and a real number $\rho$ such that the scalar curvature of $M$ satisfies the following equation

$$
\left(\operatorname{scal}_{g}-\rho\right) g=\frac{1}{2} \boldsymbol{L}_{X} g,
$$

where $\boldsymbol{L}_{X}$ is the Lie derivative along to $X$, then $(M, g)$ is called a Yamabe soliton. The Yamabe soliton is said to be shrinking, steady or expanding if $\rho>0, \rho=0$ or $\rho<0$, respectively. If $X=\operatorname{grad} \varphi$ for some smooth function $\varphi$ on $M$, then we say that $(M, g, \operatorname{grad} \varphi, \rho)$ is called a gradient Yamabe soliton with potential function $\varphi$. Thus, the equation (1.1) turns into

$$
\left(\operatorname{scal}_{g}-\rho\right) g=H e s s \varphi,
$$

where Hess $\varphi$ denotes the Hessian of $\varphi$. When $\varphi$ is constant, a gradient Yamabe soliton turns into a trivial Yamabe soliton. If $\rho$ is a differentiable function on $M$ then we obtain an almost Yamabe soliton. In particular, for gradient vector field, we obtain an almost gradient Yamabe soliton [1].

In [11], Ma and Miquel studied the scalar curvature of Yamabe solitons. In [2], Cao, Sun and Zhang studied every complete nontrivial gradient Yamabe soliton admits a special global warped product structure with a one-dimensional base. In [7], He studied a complete gradient steady Yamabe soliton on warped product. In [12], Neto and Tenenblat studied gradient Yamabe solitons conformal to an $n$-dimensional pseudo-Euclidean space. In [16], Tokura, Adriano, Pina and Barboza studied gradient Yamabe soliton on warped product manifolds with limited warping function, and for the compact base. For other developments about Ricci solitons and gradient Ricci soliton; [3], [5], [8], [9], [10], [13], [17]. Motivated by the above studies, in the present paper, we consider gradient Yamabe solitons on multiply warped product manifolds. We find the necessary and sufficient conditions for multiply warped product manifolds to be gradient Yamabe solitons.

\section{Preliminaries}

Let $\left(B, g_{B}\right)$ and $\left(F_{i}, g_{F_{i}}\right)$ be $r$ and $s_{i}$ dimensional semi-Riemannian manifolds, respectively, where $i \in$ $\{1,2, \ldots, m\}$ and also $M=B \times F_{1} \times F_{2} \times \ldots \times F_{m}$ be an $n=r+t$ dimensional semi-Riemannian manifold, where $t=\sum_{i=1}^{m} s_{i}$. Let $b_{i}: B \rightarrow(0, \infty)$ be positive smooth functions for $1 \leq i \leq m$. The product manifold $M=$ 
$B \times_{b_{1}} F_{1} \times_{b_{2}} F_{2} \times \ldots \times_{b_{m}} F_{m}$ endowed with the metric tensor

$$
\bar{g}=\pi^{*}\left(g_{B}\right) \oplus\left(b_{1} \circ \pi\right)^{2} \sigma_{1}^{*}\left(g_{F_{1}}\right) \oplus \ldots \oplus\left(b_{m} \circ \pi\right)^{2} \sigma_{m}^{*}\left(g_{F_{m}}\right),
$$

where $\pi$ and $\sigma_{i}$ are the natural projection on $B$ and $F_{i}$, respectively, is called the multiply warped product. The functions $b_{i}: B \rightarrow(0, \infty)$ are called warping functions and each manifolds $\left(F_{i}, g_{F_{i}}\right)$ and the manifold $\left(B, g_{B}\right)$ are called fiber manifolds and the base manifold of the multiply warped product, respectively for $1 \leq i \leq m$ ([4], [14], [15]). We shall denote $s c a l, s c a l_{B}, s_{c a l} F_{i}=\lambda_{F_{i}}$ the scalar curvatures of the $M, B$ and $F_{i}$, respectively.

Now, we give the following lemmas:

Lemma 2.1. [14] Let $M=B \times_{b_{1}} F_{1} \times_{b_{2}} F_{2} \times \ldots \times_{b_{m}} F_{m}$ be a multiply warped product with metric $\bar{g}=g_{B} \oplus b_{1}^{2} g_{F_{1}} \oplus$ $\ldots \oplus b_{m}^{2} g_{F_{m}}$. If $X, Y \in \chi(B)$ and $V \in \chi\left(F_{i}\right), W \in \chi\left(F_{j}\right)$, then

i) $\nabla_{X} Y$ is the lift of ${ }^{B} \nabla_{X} Y$ on $B$,

ii) $\nabla_{X} V=\nabla_{V} X=\frac{X\left(b_{i}\right)}{b_{i}} V$,

iii) $\nabla_{V} W=\left\{\begin{array}{cc}0 & \text { if } i \neq j, \\ F_{i} \nabla_{V} W-\left(\frac{g(V, W)}{b_{i}}\right) \operatorname{grad}_{B}\left(b_{i}\right) & \text { if } i=j .\end{array}\right.$

Lemma 2.2. [14]Let $M=B \times_{b_{1}} F_{1} \times_{b_{2}} F_{2} \times \ldots \times_{b_{m}} F_{m}$ be a multiply warped product with metric $\bar{g}=g_{B} \oplus b_{1}^{2} g_{F_{1}} \oplus$ $\ldots \oplus b_{m}^{2} g_{F_{m}}$. Then

$$
\begin{aligned}
s c a l=s c a l_{B}- & 2 \sum_{i=1}^{m} s_{i} \frac{\Delta_{B} b_{i}}{b_{i}}+\sum_{i=1}^{m} \frac{\operatorname{scal}_{F_{i}}}{b_{i}^{2}}-\sum_{i=1}^{m} s_{i}\left(s_{i}-1\right) \frac{\left\|\operatorname{grad}_{B} b_{i}\right\|^{2}}{b_{i}^{2}} \\
& -\sum_{i=1}^{m} \sum_{i=1, l \neq i}^{m} s_{i} s_{l} \frac{g_{B}\left(\operatorname{grad}_{B} b_{i}, \operatorname{grad}_{B} b_{l}\right)}{b_{i} b_{l}} .
\end{aligned}
$$

\section{The gradient Yamabe soliton on multiply warped product}

Let $\varphi$ be a potential function of a gradient Yamabe soliton on multiply warped product $(M, \bar{g})$.

Proposition 3.1. Let $M=B \times_{b_{1}} F_{1} \times_{b_{2}} F_{2} \times \ldots \times_{b_{m}} F_{m}$ be a multiply warped product with $\bar{g}=g_{B} \oplus b_{1}^{2} g_{F_{1}} \oplus \ldots \oplus$ $b_{m}^{2} g_{F_{m}}$. If the multiply warped product $(M, \bar{g})$ is a gradient Yamabe soliton with potential function $\varphi: M \longrightarrow \mathbb{R}$, and there exists a pair of orthogonal vectors $\left(X_{\imath}, X_{j}\right)$ of the base, such that $\operatorname{Hess}_{B}(\varphi)\left(X_{\imath}, X_{j}\right) \neq 0$ then the potential function $\varphi$ depends only on the base.

Proof. Assume that $M=B \times_{b_{1}} F_{1} \times_{b_{2}} F_{2} \times \ldots \times_{b_{m}} F_{m}$ is a gradient Yamabe soliton with potential function $\varphi: M \longrightarrow \mathbb{R}$. Using the equation (1.2) and Lemma 2.2, we have

$$
\begin{gathered}
\left\{\operatorname{scal}_{B}-2 \sum_{i=1}^{m} s_{i} \frac{\Delta_{B} b_{i}}{b_{i}}+\sum_{i=1}^{m} \frac{s c a l_{F_{i}}}{b_{i}^{2}}-\sum_{i=1}^{m} s_{i}\left(s_{i}-1\right) \frac{\left\|\operatorname{grad}_{B} b_{i}\right\|^{2}}{b_{i}^{2}}\right. \\
\left.-\sum_{i=1}^{m} \sum_{i=1, l \neq i}^{m} s_{i} s_{l} \frac{g_{B}\left(\operatorname{grad}_{B} b_{i}, \operatorname{grad}_{B} b_{l}\right)}{b_{i} b_{l}}-\rho\right\} g_{B}\left(X_{i}, X_{\jmath}\right)=\operatorname{Hess\varphi }\left(X_{i}, X_{\jmath}\right), \\
\left\{s c a l_{B}-2 \sum_{i=1}^{m} s_{i} \frac{\Delta_{B} b_{i}}{b_{i}}+\sum_{i=1}^{m} \frac{s c a l_{F_{i}}}{b_{i}^{2}}-\sum_{i=1}^{m} s_{i}\left(s_{i}-1\right) \frac{\left\|\operatorname{grad}_{B} b_{i}\right\|^{2}}{b_{i}^{2}}\right. \\
\left.-\sum_{i=1}^{m} \sum_{i=1, l \neq i}^{m} s_{i} s_{l} \frac{g_{B}\left(\operatorname{grad}_{B} b_{i}, \operatorname{grad}_{B} b_{l}\right)}{b_{i} b_{l}}-\rho\right\} \bar{g}\left(X_{i}, Y_{\jmath}\right)=\operatorname{Hess\varphi }\left(X_{i}, Y_{\jmath}\right), \\
\left\{\operatorname{scal}_{B}-2 \sum_{i=1}^{m} s_{i} \frac{\Delta_{B} b_{i}}{b_{i}}+\sum_{i=1}^{m} \frac{\operatorname{scal}_{F_{i}}}{b_{i}^{2}}-\sum_{i=1}^{m} s_{i}\left(s_{i}-1\right) \frac{\left\|\operatorname{grad}_{B} b_{i}\right\|^{2}}{b_{i}^{2}}\right. \\
\left.-\sum_{i=1}^{m} \sum_{i=1, l \neq i}^{m} s_{i} s_{l} \frac{g_{B}\left(\operatorname{grad}_{B} b_{i}, \operatorname{grad}_{B} b_{l}\right)}{b_{i} b_{l}}-\rho\right\}\left(\sum_{i=1}^{m} b_{i}^{2} g_{F_{i}}\left(Y_{\imath}, Y_{\jmath}\right)\right)
\end{gathered}
$$




$$
=\operatorname{Hess\varphi }\left(Y_{\imath}, Y_{\jmath}\right)
$$

where $X_{1}, X_{2}, \ldots, X_{r} \in \chi(B)$ and $Y_{1}, Y_{2}, \ldots, Y_{t} \in \chi\left(F_{i}\right)$ for $1 \leq \imath \leq r$ and $1 \leq \jmath \leq t$. Using the equation $\bar{g}\left(X_{\imath}, Y_{\jmath}\right)=$ 0 , we obtain $\operatorname{Hess} \varphi\left(X_{\imath}, Y_{\jmath}\right)=0$.

From Lemma 2.1 in [6], we can write

$$
\varphi(X, Y)=z(x)+b_{i}(x) \nu_{i}(y)
$$

where $z: B \longrightarrow \mathbb{R}$ and $v_{i}: F_{i} \longrightarrow \mathbb{R}$. Then, we have $\operatorname{Hess} \varphi\left(X_{\imath}, X_{\jmath}\right)=\operatorname{Hess}_{B} \varphi\left(X_{\imath}, X_{\jmath}\right)$. Using the last equation in (3.1), we obtain

$$
\begin{gathered}
\left\{\operatorname{scal}_{B}-2 \sum_{i=1}^{m} s_{i} \frac{\Delta_{B} b_{i}}{b_{i}}+\sum_{i=1}^{m} \frac{\operatorname{scal}_{F_{i}}}{b_{i}^{2}}-\sum_{i=1}^{m} s_{i}\left(s_{i}-1\right) \frac{\left\|g r a d_{B} b_{i}\right\|^{2}}{b_{i}^{2}}\right. \\
\left.-\sum_{i=1}^{m} \sum_{i=1, l \neq i}^{m} s_{i} s_{l} \frac{g_{B}\left(\operatorname{grad}_{B} b_{i}, \operatorname{grad}_{B} b_{l}\right)}{b_{i} b_{l}}-\rho\right\} g_{B}\left(X_{\imath}, X_{\jmath}\right)=\operatorname{Hess}_{B} \varphi\left(X_{\imath}, X_{\jmath}\right) .
\end{gathered}
$$

Substituting the equation (3.4) in (3.5), we find

$$
\begin{gathered}
\left\{s c a l_{B}-2 \sum_{i=1}^{m} s_{i} \frac{\Delta_{B} b_{i}}{b_{i}}+\sum_{i=1}^{m} \frac{s c a l_{F_{i}}}{b_{i}^{2}}-\sum_{i=1}^{m} s_{i}\left(s_{i}-1\right) \frac{\left\|\operatorname{grad}_{B} b_{i}\right\|^{2}}{b_{i}^{2}}\right. \\
\left.-\sum_{i=1}^{m} \sum_{i=1, l \neq i}^{m} s_{i} s_{l} \frac{g_{B}\left(\operatorname{grad}_{B} b_{i}, \operatorname{grad}_{B} b_{l}\right)}{b_{i} b_{l}}-\rho\right\} g_{B}\left(X_{i}, X_{\jmath}\right)=\text { Hess }_{B} z+\nu_{i} \text { Hess }_{B} b_{i} .
\end{gathered}
$$

By the use of the equation $\operatorname{Hess}_{B}(\varphi)\left(X_{\imath}, X_{\jmath}\right)=\operatorname{Hess}_{B} b_{i}\left(X_{\imath}, X_{\jmath}\right) \neq 0$, we can write

$$
\nu_{i}=-\frac{\operatorname{Hess}_{B} z\left(X_{\imath}, X_{\jmath}\right)}{\operatorname{Hess}_{B} b_{i}\left(X_{\imath}, X_{\jmath}\right)} \text {. }
$$

From the equation (3.6), we obtain that the potential function $\varphi$ depends only on the base.

From Proposition 3.1, we consider a multiply warped product gradient Yamabe soliton $\left(M=B \times_{b_{1}} F_{1} \times_{b_{2}} F_{2} \times \ldots \times_{b_{m}} F_{m}, \bar{g}, \operatorname{grad} \varphi, \rho\right)$, splitting of the form $\varphi$

$$
\varphi(X, Y)=\varphi_{0}(X)+\sum_{k=1}^{m} \varphi_{k}(Y),
$$

where $\varphi_{0} \in C^{\infty}(B)$ and $\varphi_{k} \in C^{\infty}\left(F_{i}\right)$.

Now, we give the following Theorem:

Theorem 3.1. Let $\left(M=B \times_{b_{1}} F_{1} \times_{b_{2}} F_{2} \times \ldots \times_{b_{m}} F_{m}, \bar{g}, \operatorname{grad} \varphi, \rho\right)$ be a gradient Yamabe soliton on multiply warped product given by $\varphi(X, Y)=\varphi_{0}(X)+\sum_{k=1}^{m} \varphi_{k}(Y)$, then one of the following cases occurs

(1) $M$ is the Riemannian product between a trivial gradient Yamabe soliton and $m$ gradient Yamabe solitons,

(2) $M$ is the Riemannian product between $(m+1)$ gradient Yamabe solitons,

(3) $M$ is the multiply warped product between an almost gradient Yamabe soliton and $m$ trivial gradient Yamabe solitons.

Proof. Assume that $M=B \times_{b_{1}} F_{1} \times_{b_{2}} F_{2} \times \ldots \times_{b_{m}} F_{m}$ is a gradient Yamabe soliton with potential function $\varphi(X, Y)=\varphi_{0}(X)+\sum_{k=1}^{m} \varphi_{k}(Y)$. For $X_{1}, X_{2}, \ldots, X_{r} \in \chi(B)$ and $Y_{1}, Y_{2}, \ldots, Y_{t} \in \chi\left(F_{i}\right)$ where $1 \leq \imath \leq r$ and $1 \leq \jmath \leq t$, we have Hess $(\varphi)\left(X_{\imath}, Y_{\jmath}\right)=0$. Using part ii) of Lemma 2.1, we find

$$
\operatorname{Hess}(\varphi)\left(X_{\imath}, Y_{\jmath}\right)=X_{\imath}\left(Y_{\jmath}(\varphi)\right)-\frac{X_{\imath}\left(b_{i}\right)}{b_{i}} Y_{\jmath}(\varphi)=0 .
$$

Using the equation (3.7) in (3.8), we obtain

$$
X_{\imath}\left(Y_{\jmath}(\varphi)\right)-\frac{X_{\imath}\left(b_{i}\right)}{b_{i}} Y_{\jmath}(\varphi)=0-\sum_{k=1}^{m} \frac{X_{\imath}\left(b_{i}\right)}{b_{i}} Y_{\jmath}\left(\varphi_{k}\right)=0 .
$$


Then, $b_{i}$ are constant or $\varphi(X, Y)=\varphi_{0}(X)+$ constant. We investigate the proof in three cases:

(1) Let $b_{i}$ be constant or $\varphi(X, Y)=\varphi_{0}(X)+$ constant. In this case, $M=B \times_{b_{1}} F_{1} \times_{b_{2}} F_{2} \times \ldots \times_{b_{m}} F_{m}$ is a Riemannian product and we have

$$
\begin{gathered}
\left(\operatorname{scal}_{B}+\sum_{i=1}^{m} \frac{\operatorname{scal}_{F_{i}}}{b_{i}^{2}}-\rho\right) g_{B}\left(X_{\imath}, X_{\jmath}\right)=\operatorname{Hess}_{B} \varphi_{0}\left(X_{\imath}, X_{\jmath}\right), \\
\left(\operatorname{scal}_{B}+\sum_{i=1}^{m} \frac{\operatorname{scal}_{F_{i}}}{b_{i}^{2}}-\rho\right) \bar{g}\left(X_{\imath}, Y_{\jmath}\right)=\operatorname{Hess\varphi }\left(X_{\imath}, Y_{\jmath}\right)=0 \\
\left(\operatorname{scal}_{B}+\sum_{i=1}^{m} \frac{\operatorname{scal}_{F_{i}}}{b_{i}^{2}}-\rho\right)\left(\sum_{i=1}^{m} b_{i}^{2} g_{F_{i}}\left(Y_{\imath}, Y_{\jmath}\right)\right)=\operatorname{Hess\varphi }\left(Y_{\imath}, Y_{\jmath}\right) .
\end{gathered}
$$

From part iii) of Lemma 2.1, we can write

$$
\begin{gathered}
\operatorname{Hess\varphi }\left(Y_{\imath}, Y_{\jmath}\right)=Y_{\imath}\left(Y_{\jmath}(\varphi)\right)-\left({ }^{M} \nabla_{Y_{\imath}} Y_{\jmath}\right) \varphi \\
=Y_{\imath}\left(Y_{\jmath}(\varphi)\right)+\frac{g\left(Y_{\imath}, Y_{\jmath}\right)}{b_{i}} \operatorname{grad}_{B}\left(b_{i}\right)(\varphi)-{ }^{F_{i}} \nabla_{Y_{\imath}} Y_{\jmath}(\varphi) \\
=\operatorname{Hess}_{F_{i}} \varphi_{k}\left(Y_{\imath}, Y_{\jmath}\right)+\frac{\sum_{i=1}^{m} b_{i}^{2} g_{F_{i}}\left(Y_{\imath}, Y_{\jmath}\right)}{b_{i}} \operatorname{grad}_{B}\left(b_{i}\right)(\varphi) .
\end{gathered}
$$

Using the equation (3.13) in (3.12), we obtain

$$
\begin{aligned}
& \left(\operatorname{scal}_{B}+\sum_{i=1}^{m} \frac{s c a l_{F_{i}}}{b_{i}^{2}}-\rho\right)\left(\sum_{i=1}^{m} b_{i}^{2} g_{F_{i}}\left(Y_{\imath}, Y_{\jmath}\right)\right) \\
= & \operatorname{Hess}_{F_{i}} \varphi_{k}\left(Y_{i}, Y_{\jmath}\right)+\frac{\sum_{i=1}^{m} b_{i}^{2} g_{F_{i}}\left(Y_{\imath}, Y_{\jmath}\right)}{b_{i}} \operatorname{grad}_{B}\left(b_{i}\right)(\varphi) .
\end{aligned}
$$

Since $\operatorname{scal}_{F_{i}}$ is a constant on $B$, we find from (3.10) that $B$ is a gradient Yamabe soliton of the form $\left(B, g_{B}, \operatorname{grad} \varphi_{0},-\sum_{i=1}^{m} \frac{\text { scal }_{F_{i}}}{b_{i}^{2}}+\rho\right)$. Moreover, since $\varphi(X, Y)=\varphi_{0}(X)+$ constant, we have from the equation (3.14) that $F_{i}$ are trivial gradient Yamabe solitons of the form $\left(F_{i}, g_{F_{i}}, \operatorname{grad} 0,-\left(\sum_{i=1}^{m} b_{i}^{2}\right) s c a l_{B}+\left(\sum_{i=1}^{m} b_{i}^{2}\right) \rho\right)$. This proves the first assertion of the theorem.

(2) Let $b_{i}$ be constant or $\varphi(X, Y)=\varphi_{0}(X)+\sum_{k=1}^{m} \varphi_{k}$, where $\varphi_{k}$ is not necessarily constant. In this case, $M=B \times_{b_{1}} F_{1} \times_{b_{2}} F_{2} \times \ldots \times_{b_{m}} F_{m}$ is a Riemannian product and we have

$$
\begin{gathered}
\left(\operatorname{scal}_{B}+\sum_{i=1}^{m} \frac{\operatorname{scal}_{F_{i}}}{b_{i}^{2}}-\rho\right) g_{B}\left(X_{\imath}, X_{\jmath}\right)=\operatorname{Hess}_{B} \varphi_{0}\left(X_{\imath}, X_{\jmath}\right), \\
\left(\operatorname{scal}_{B}+\sum_{i=1}^{m} \frac{\operatorname{scal}_{F_{i}}}{b_{i}^{2}}-\rho\right) \bar{g}\left(X_{\imath}, Y_{\jmath}\right)=\operatorname{Hess} \varphi\left(X_{\imath}, Y_{\jmath}\right)=0, \\
\left(\operatorname{scal}_{B}+\sum_{i=1}^{m} \frac{s c a l_{F_{i}}}{b_{i}^{2}}-\rho\right)\left(\sum_{i=1}^{m} b_{i}^{2} g_{F_{i}}\left(Y_{\imath}, Y_{\jmath}\right)\right)=\operatorname{Hess}_{F_{i}} \varphi_{k}\left(Y_{\imath}, Y_{\jmath}\right) \\
+\frac{\sum_{i=1}^{m} b_{i}^{2} g_{F_{i}}\left(Y_{\imath}, Y_{\jmath}\right)}{b_{i}} \operatorname{grad}_{B}\left(b_{i}\right)(\varphi) .
\end{gathered}
$$


Since $\operatorname{scal}_{F_{i}}$ is a constant on $B$, we find from (3.15) that $B$ is a gradient Yamabe soliton of the form $\left(B, g_{B}, \operatorname{grad} \varphi_{0},-\sum_{i=1}^{m} \frac{\operatorname{scal}_{F_{i}}}{b_{i}^{2}}+\rho\right)$. From the equation (3.17), we obtain that $F_{i}$ are gradient Yamabe solitons of the form $\left(F_{i}, g_{F_{i}}, \operatorname{grad} \varphi_{k},-\left(\sum_{i=1}^{m} b_{i}^{2}\right) s c a l_{B}+\left(\sum_{i=1}^{m} b_{i}^{2}\right) \rho\right)$. This proves the second assertion of the theorem.

(3) Let $b_{i}$ be non constant or $\varphi(X, Y)=\varphi_{0}(X)+$ constant. In this case, we can write

$$
\begin{gathered}
\left\{\operatorname{scal}_{B}-2 \sum_{i=1}^{m} s_{i} \frac{\Delta_{B} b_{i}}{b_{i}}+\sum_{i=1}^{m} \frac{s c a l_{F_{i}}}{b_{i}^{2}}-\sum_{i=1}^{m} s_{i}\left(s_{i}-1\right) \frac{\left\|\operatorname{grad}_{B} b_{i}\right\|^{2}}{b_{i}^{2}}\right. \\
\left.-\sum_{i=1}^{m} \sum_{i=1, l \neq i}^{m} s_{i} s_{l} \frac{g_{B}\left(\operatorname{grad}_{B} b_{i}, \operatorname{grad}_{B} b_{l}\right)}{b_{i} b_{l}}-\rho\right\} g_{B}\left(X_{\imath}, X_{\jmath}\right)=\operatorname{Hess}_{B} \varphi_{0}\left(X_{i}, X_{\jmath}\right), \\
\left\{s c a l_{B}-2 \sum_{i=1}^{m} s_{i} \frac{\Delta_{B} b_{i}}{b_{i}}+\sum_{i=1}^{m} \frac{s c a l_{F_{i}}}{b_{i}^{2}}-\sum_{i=1}^{m} s_{i}\left(s_{i}-1\right) \frac{\left\|\operatorname{grad}_{B} b_{i}\right\|^{2}}{b_{i}^{2}}\right. \\
\left.-\sum_{i=1}^{m} \sum_{i=1, l \neq i}^{m} s_{i} s_{l} \frac{g_{B}\left(\operatorname{grad}_{B} b_{i}, \operatorname{grad}_{B} b_{l}\right)}{b_{i} b_{l}}-\rho\right\} \bar{g}\left(X_{i}, Y_{\jmath}\right)=\operatorname{Hess\varphi }\left(X_{i}, Y_{\jmath}\right)=0, \\
\left\{s c a l_{B}-2 \sum_{i=1}^{m} s_{i} \frac{\Delta_{B} b_{i}}{b_{i}}+\sum_{i=1}^{m} \frac{\operatorname{scal}_{F_{i}}}{b_{i}^{2}}-\sum_{i=1}^{m} s_{i}\left(s_{i}-1\right) \frac{\left\|\operatorname{grad}_{B} b_{i}\right\|^{2}}{b_{i}^{2}}\right. \\
\left.-\sum_{i=1}^{m} \sum_{i=1, l \neq i}^{m} s_{i} s_{l} \frac{g_{B}\left(\operatorname{grad}_{B} b_{i}, \operatorname{grad}_{B} b_{l}\right)}{b_{i} b_{l}}-\rho\right\}\left(\sum_{i=1}^{m} b_{i}^{2} g_{F_{i}}\left(Y_{i}, Y_{\jmath}\right)\right)=\operatorname{Hess\varphi }\left(Y_{\imath}, Y_{\jmath}\right) .
\end{gathered}
$$

From the equation (3.13) and $\varphi(X, Y)=\varphi_{0}(X)+$ constant, we find

$$
\begin{gathered}
\left\{\operatorname{scal}_{B}-2 \sum_{i=1}^{m} s_{i} \frac{\Delta_{B} b_{i}}{b_{i}}+\sum_{i=1}^{m} \frac{\operatorname{scal}_{F_{i}}}{b_{i}^{2}}-\sum_{i=1}^{m} s_{i}\left(s_{i}-1\right) \frac{\left\|\operatorname{grad}_{B} b_{i}\right\|^{2}}{b_{i}^{2}}\right. \\
\left.-\sum_{i=1}^{m} \sum_{i=1, l \neq i}^{m} s_{i} s_{l} \frac{g_{B}\left(\operatorname{grad}_{B} b_{i}, \operatorname{grad}_{B} b_{l}\right)}{b_{i} b_{l}}-\rho\right\}\left(\sum_{i=1}^{m} b_{i}^{2} g_{F_{i}}\left(Y_{\imath}, Y_{\jmath}\right)\right) \\
=\frac{\sum_{i=1}^{m} b_{i}^{2} g_{F_{i}}\left(Y_{i}, Y_{\jmath}\right)}{b_{i}} \operatorname{grad}_{B}\left(b_{i}\right)\left(\varphi_{0}\right) .
\end{gathered}
$$

Since $b_{i}$ are positive for $1 \leq i \leq m$, by equation (3.21) we obtain

$$
\left(\sum_{i=1}^{m} \operatorname{scal}_{F_{i}}-\psi\right)\left(\sum_{i=1}^{m} b_{i}^{2} g_{F_{i}}\left(Y_{i}, Y_{\jmath}\right)\right)=0
$$

where

$$
\begin{gathered}
\psi=\left(\sum_{i=1}^{m} b_{i}^{2}\right)\left(\operatorname{scal}_{B}-2 \sum_{i=1}^{m} s_{i} \frac{\Delta_{B} b_{i}}{b_{i}}-\sum_{i=1}^{m} s_{i}\left(s_{i}-1\right) \frac{\left\|g r a d_{B} b_{i}\right\|^{2}}{b_{i}^{2}}\right. \\
\left.-\sum_{i=1}^{m} \sum_{i=1, l \neq i}^{m} s_{i} s_{l} \frac{g_{B}\left(\operatorname{grad}_{B} b_{i}, \operatorname{grad}_{B} b_{l}\right)}{b_{i} b_{l}}-\frac{\operatorname{grad}_{B}\left(b_{i}\right)\left(\varphi_{1}\right)}{b_{i}}-\rho\right) .
\end{gathered}
$$

Since $\psi$ depend only on $\mathrm{B}$, we have that $\psi$ is constant on $F_{i}$, then by equation (3.21), we have that $F_{i}$ are a trivial gradient Yamabe solitons. Moreover, by equation (3.18) we have that $\left(B, g_{B}\right)$ is a gradient almost Yamabe soliton of the form

$$
\left(B, g_{B}, \operatorname{grad} \varphi_{0},-\left[\sum_{i=1}^{m} \frac{s c a l_{F_{i}}}{b_{i}^{2}}-2 \sum_{i=1}^{m} s_{i} \frac{\Delta_{B} b_{i}}{b_{i}}-\sum_{i=1}^{m} s_{i}\left(s_{i}-1\right) \frac{\left\|\operatorname{grad}_{B} b_{i}\right\|^{2}}{b_{i}^{2}}\right.\right.
$$




$$
\left.\left.-\sum_{i=1}^{m} \sum_{i=1, l \neq i}^{m} s_{i} s_{l} \frac{g_{B}\left(\operatorname{grad}_{B} b_{i}, \operatorname{grad}_{B} b_{l}\right)}{b_{i} b_{l}}-\rho\right]\right) .
$$

This proves the third assertion of the theorem.

The above theorem denotes us that if we take the potential function depending only on the base then the fibers $F_{i}$ are constant scalar curvature. Then, we will take a gradient Yamabe soliton with potential function of the form $\varphi(X, Y)=\varphi_{0}(X)+$ constant on a multiply warped product, the base conformal to an $r$-dimensional pseudo-Euclidean space, and the fibers chosen to be scalar constant spaces. Let $\left(\mathbb{R}^{r}, g_{\mathbb{R}}\right)$ be the pseudo-Euclidean space, $r \geq 3$ with coordinates $X=\left(X_{1}, \ldots, X_{r}\right), g_{\imath \jmath}=\delta_{\imath \jmath} \epsilon_{\imath \jmath}$ and let $M=\left(\mathbb{R}^{r}, \bar{g}\right) \times_{b_{i}} F_{i}^{s_{i}}$ be a multiply warped product where $\widetilde{g}=\frac{1}{\phi^{2}} g_{\mathbb{R}}, F_{i}$ a semi-Riemannian scalar constant manifolds with curvatures $\lambda_{F_{i}}, t=\sum_{i=1}^{m} s_{i} \geq 1, b_{i}, \phi, \varphi: \mathbb{R}^{r} \rightarrow \mathbb{R}$, smooth functions, and $b_{i}$ are positive functions. Then, we obtain necessary and sufficient conditions for the multiply warped product to be a gradient Yamabe soliton.

Now, we give the following Theorem:

Theorem 3.2. Let $\left(\mathbb{R}^{r}, g_{\mathbb{R}}\right)$ be a pseudo-Euclidean space, $r \geq 3$ with coordinates, $X=\left(X_{1}, \ldots, X_{r}\right)$ and $g_{\imath \jmath}=\delta_{\imath \jmath} \epsilon_{\imath \jmath}$, let $M=\left(\mathbb{R}^{r}, \widetilde{g}\right) \times_{b_{1}} F_{1} \times_{b_{2}} F_{2} \times \ldots \times_{b_{m}} F_{m}$, be a multiply warped product where $\widetilde{g}=\frac{1}{\phi^{2}} g_{\mathbb{R}}$ and $F_{i}$ semi-Riemannian scalar-constant manifolds with curvatures $\lambda_{F_{i}}, t=\sum_{i=1}^{m} s_{i} \geq 1, b_{i}, \phi, \varphi: \mathbb{R}^{r} \rightarrow \mathbb{R}$, smooth functions, and $b_{i}$ are positive functions. Then, the multiply warped product $(M, \bar{g})$ is a gradient Yamabe soliton with potential function $\varphi$ if and only if the functions $b_{i}, \phi, \varphi$ satisfy

$$
\begin{aligned}
& \varphi_{x_{\imath} x_{\jmath}}+\frac{\phi_{x_{\imath}}}{\phi} \varphi_{x_{\jmath}}+\frac{\phi_{x_{\jmath}}}{\phi} \varphi_{x_{\imath}}=0 \quad \imath \neq \jmath, \\
& \left\{(r-1)\left(2 \phi \sum_{k} \epsilon_{k} \phi_{x_{k} x_{k}}-r \sum_{k} \epsilon_{k} \phi_{x_{k}}^{2}\right)\right. \\
& -2 \sum_{i=1}^{m} s_{i} \frac{\phi^{2} \sum_{k} \epsilon_{k}\left(b_{i}\right)_{x_{k} x_{k}}-(n-2) \phi \sum_{k} \epsilon_{k} \phi_{x_{k}}\left(b_{i}\right)_{x_{k}}}{b_{i}} \\
& +\sum_{i=1}^{m} \frac{\lambda_{F_{i}}}{b_{i}^{2}}-\sum_{i=1}^{m} s_{i}\left(s_{i}-1\right) \frac{\left(\phi^{2} \sum_{k} \epsilon_{k}\left(b_{i}^{2}\right)_{x_{k}}\right)}{b_{i}^{2}} \\
& \left.-\sum_{i=1}^{m} \sum_{i=1, l \neq i}^{m} s_{i} s_{l} \frac{\left(\phi^{2} \sum_{k=1}^{r} \epsilon_{k}\left(b_{i}\right)_{x_{k}}\left(b_{l}\right)_{x_{k}}\right)}{b_{i} b_{l}}-\rho\right\} \frac{\epsilon_{i}}{\phi^{2}} \\
& =\varphi_{x_{\imath} x_{\imath}}+2 \frac{\phi_{x_{\imath}}}{\phi} \varphi_{x_{\imath}}-\epsilon_{i} \sum_{k} \epsilon_{k} \frac{\phi_{x_{k}}}{\phi} \varphi_{x_{k}}, \\
& \left\{(r-1)\left(2 \phi \sum_{k} \epsilon_{k} \phi_{x_{k} x_{k}}-r \sum_{k} \epsilon_{k} \phi_{x_{k}}^{2}\right)\right. \\
& -2 \sum_{i=1}^{m} s_{i} \frac{\phi^{2} \sum_{k} \epsilon_{k}\left(b_{i}\right)_{x_{k} x_{k}}-(n-2) \phi \sum_{k} \epsilon_{k} \phi_{x_{k}}\left(b_{i}\right)_{x_{k}}}{b_{i}} \\
& +\sum_{i=1}^{m} \frac{\lambda_{F_{i}}}{b_{i}^{2}}-\sum_{i=1}^{m} s_{i}\left(s_{i}-1\right) \frac{\left(\phi^{2} \sum_{k} \epsilon_{k}\left(b_{i}^{2}\right)_{x_{k}}\right)}{b_{i}^{2}} \\
& \left.-\sum_{i=1}^{m} \sum_{i=1, l \neq i}^{m} s_{i} s_{l} \frac{\left(\phi^{2} \sum_{k} \epsilon_{k}\left(b_{i}\right)_{x_{k}}\left(b_{l}\right)_{x_{k}}\right)}{b_{i} b_{l}}-\rho\right\}=\frac{\phi^{2}}{b_{i}} \sum_{k} \epsilon_{k} \phi_{x_{k}}\left(b_{i}\right)_{x_{k}} .
\end{aligned}
$$


Proof. Assume that $M=\left(\mathbb{R}^{r}, \widetilde{g}\right) \times_{b_{1}} F_{1} \times_{b_{2}} F_{2} \times \ldots \times_{b_{m}} F_{m}$ is multiply warped product with gradient Yamabe soliton structure. So, we have

$$
\left(\operatorname{scal}_{\bar{g}}-\rho\right) \bar{g}=\operatorname{Hess}_{\bar{g}} \varphi .
$$

For $X_{1}, X_{2}, \ldots, X_{r} \in \chi(B)$ and $Y_{1}, Y_{2}, \ldots, Y_{t} \in \chi\left(F_{i}\right)$, we can write

$$
\begin{aligned}
& \left\{\text { scal }_{\widetilde{g}}-2 \sum_{i=1}^{m} s_{i} \frac{\Delta_{\widetilde{g}} b_{i}}{b_{i}}+\sum_{i=1}^{m} \frac{s c a l_{F_{i}}}{b_{i}^{2}}-\sum_{i=1}^{m} s_{i}\left(s_{i}-1\right) \frac{\left\|\operatorname{grad}_{\widetilde{g}} b_{i}\right\|^{2}}{b_{i}^{2}}\right. \\
& \left.-\sum_{i=1}^{m} \sum_{i=1, l \neq i}^{m} s_{i} s_{l} \frac{\widetilde{g}\left(\operatorname{grad}_{\widetilde{g}} b_{i}, \operatorname{grad}_{\widetilde{g}} b_{l}\right)}{b_{i} b_{l}}-\rho\right\} \widetilde{g}\left(X_{\imath}, X_{\jmath}\right)=\operatorname{Hess}_{\bar{g}} \varphi\left(X_{\imath}, X_{\jmath}\right) \text {, } \\
& \left\{\operatorname{scal}_{\widetilde{g}}-2 \sum_{i=1}^{m} s_{i} \frac{\Delta_{\widetilde{g}} b_{i}}{b_{i}}+\sum_{i=1}^{m} \frac{\operatorname{scal}_{F_{i}}}{b_{i}^{2}}-\sum_{i=1}^{m} s_{i}\left(s_{i}-1\right) \frac{\left\|\operatorname{grad}_{\widetilde{g}} b_{i}\right\|^{2}}{b_{i}^{2}}\right. \\
& \left.-\sum_{i=1}^{m} \sum_{i=1, l \neq i}^{m} s_{i} s_{l} \frac{\widetilde{g}\left(\operatorname{grad}_{\widetilde{g}} b_{i}, \operatorname{grad}_{\widetilde{g}} b_{l}\right)}{b_{i} b_{l}}-\rho\right\} \bar{g}\left(X_{\imath}, Y_{\jmath}\right)=\operatorname{Hess}_{\bar{g}} \varphi\left(X_{\imath}, Y_{\jmath}\right)=0, \\
& \left\{\operatorname{scal}_{\widetilde{g}}-2 \sum_{i=1}^{m} s_{i} \frac{\Delta_{\widetilde{g}} b_{i}}{b_{i}}+\sum_{i=1}^{m} \frac{s c a l_{F_{i}}}{b_{i}^{2}}-\sum_{i=1}^{m} s_{i}\left(s_{i}-1\right) \frac{\left\|\operatorname{grad} d_{\widetilde{g}} b_{i}\right\|^{2}}{b_{i}^{2}}\right. \\
& \left.-\sum_{i=1}^{m} \sum_{i=1, l \neq i}^{m} s_{i} s_{l} \frac{\widetilde{g}\left(\operatorname{grad}_{\widetilde{g}} b_{i}, \operatorname{grad}_{\widetilde{g}} b_{l}\right)}{b_{i} b_{l}}-\rho\right\}\left(\sum_{i=1}^{m} b_{i}^{2} g_{F_{i}}\left(Y_{\imath}, Y_{\jmath}\right)\right)=\operatorname{Hess}_{\bar{g}} \varphi\left(Y_{i}, Y_{\jmath}\right) .
\end{aligned}
$$

It is well known that for the conformal metric $\widetilde{g}=\frac{1}{\phi^{2}} g_{\mathbb{R}}$, the Christofel symbol is given by

$$
\bar{\Gamma}_{\imath \jmath}^{k}=0, \bar{\Gamma}_{\imath \jmath}^{\imath}=-\frac{\phi_{x_{\jmath}}}{\phi}, \bar{\Gamma}_{\imath \imath}^{k}=\epsilon_{\imath} \epsilon_{k} \frac{\phi_{x_{k}}}{\phi}, \bar{\Gamma}_{\imath \imath}^{\imath}=-\frac{\phi_{x_{\imath}}}{\phi} .
$$

Then we get

$$
\begin{gathered}
\operatorname{Hess}_{\bar{g}} \varphi_{\imath \jmath}=\varphi_{x_{\imath} x_{\jmath}}+\frac{\phi_{x_{\imath}}}{\phi} \varphi_{x_{\jmath}}+\frac{\phi_{x_{\jmath}}}{\phi} \varphi_{x_{\imath}}, \quad \imath \neq \jmath . \\
\operatorname{Hess}_{\bar{g}} \varphi_{\imath \imath}=\varphi_{x_{\imath} x_{\imath}}+2 \frac{\phi_{x_{\imath}}}{\phi} \varphi_{x_{\imath}}-\epsilon_{\imath} \sum_{k} \epsilon_{k} \frac{\phi_{x_{k}}}{\phi} \varphi_{x_{k}}, \quad \imath=\jmath .
\end{gathered}
$$

From [16], the Ricci curvature is given by

$$
\operatorname{Ric}_{\widetilde{g}}=\frac{1}{\phi^{2}}\left\{(r-2) \phi H e s s_{g_{\mathbb{R}}} \phi+\left[\phi \Delta_{g_{\mathbb{R}}} \phi-(r-1)\left|\nabla_{g_{\mathbb{R}}} \phi\right|^{2}\right] g_{\mathbb{R}}\right\} .
$$

Thus, we have the scalar curvature on conformal metric

$$
s c a l_{\widetilde{g}}=(r-1)\left(2 \phi \Delta_{g_{\mathbb{R}}} \phi-r\left|\nabla_{g_{\mathbb{R}}} \phi\right|^{2}\right)=(r-1)\left(2 \phi \sum_{k} \epsilon_{k} \phi_{x_{k} x_{k}}-r \sum_{k} \epsilon_{k} \phi_{x_{k}}^{2}\right) .
$$

Since $\varphi: \mathbb{R}^{r} \rightarrow \mathbb{R}$, we obtain

$$
\operatorname{Hess}_{\bar{g}} \varphi\left(X_{\imath}, X_{\jmath}\right)=\operatorname{Hess}_{\widetilde{g}} \varphi\left(X_{\imath}, X_{\jmath}\right), \forall \imath, \jmath
$$

On the other hand, we have

$$
\left\{\begin{array}{c}
\operatorname{scal}_{F_{i}} g_{F_{i}}=\lambda_{F_{i}} g_{F_{i}} \\
\bar{g}\left(Y_{i}, Y_{\jmath}\right)=\sum_{i=1}^{m} b_{i}^{2} g_{F_{i}}\left(Y_{i}, Y_{\jmath}\right) \\
\Delta_{\widetilde{g}} b_{i}=\phi^{2} \sum_{k} \epsilon_{k}\left(b_{i}\right)_{x_{k} x_{k}}-(n-2) \phi \sum_{k} \epsilon_{k} \phi_{x_{k}}\left(b_{i}\right)_{x_{k}} \\
\sum_{i=1}^{m} \bar{g}\left(\operatorname{grad}_{\widetilde{g}} b_{i}, \operatorname{grad}_{\widetilde{g}} b_{i}\right)=\phi^{2} \sum_{i=1}^{m} \sum_{k} \epsilon_{k}\left(b_{i}^{2}\right)_{x_{k}} \\
\sum_{i=1}^{m} \sum_{i=1, l \neq i}^{m} \bar{g}\left(\operatorname{grad}_{\widetilde{g}} b_{i}, \operatorname{grad}_{\widetilde{g}} b_{l}\right)=\phi^{2} \sum_{i=1}^{m} \sum_{i=1, l \neq i}^{m} \sum_{k} \epsilon_{k}\left(b_{i}\right)_{x_{k}}\left(b_{l}\right)_{x_{k}} .
\end{array}\right.
$$


Substituting the equations the equations (3.30), (3.31), (3.33) in equation (3.26), we find

$$
\begin{gathered}
\left\{(r-1)\left(2 \phi \sum_{k} \epsilon_{k} \phi_{x_{k} x_{k}}-r \sum_{k} \epsilon_{k} \phi_{x_{k}}^{2}\right)\right. \\
-2 \sum_{i=1}^{m} s_{i} \frac{\phi^{2} \sum_{k} \epsilon_{k}\left(b_{i}\right)_{x_{k} x_{k}}-(n-2) \phi \sum_{k} \epsilon_{k} \phi_{x_{k}}\left(b_{i}\right)_{x_{k}}}{b_{i}} \\
+\sum_{i=1}^{m} \frac{\lambda_{F_{i}}}{b_{i}^{2}}-\sum_{i=1}^{m} s_{i}\left(s_{i}-1\right) \frac{\left(\phi^{2} \sum_{k} \epsilon_{k}\left(b_{i}^{2}\right)_{x_{k}}\right)}{b_{i}^{2}} \\
\left.-\sum_{i=1}^{m} \sum_{i=1, l \neq i}^{m} s_{i} s_{l} \frac{\left(\phi^{2} \sum_{k} \epsilon_{k}\left(b_{i}\right)_{x_{k}}\left(b_{l}\right)_{x_{k}}\right)}{b_{i} b_{l}}-\rho\right\} \frac{\epsilon_{l}}{\phi^{2}} \\
=\varphi_{x_{\imath} x_{\imath}}+2 \frac{\phi_{x_{\imath}}}{\phi} \varphi_{x_{\imath}}-\epsilon_{i} \sum_{k} \epsilon_{k} \frac{\phi_{x_{k}}}{\phi} \varphi_{x_{k}} .
\end{gathered}
$$

The last equation is the equation (3.23). Similary, replacing the equation (3.29) into the equation (3.27), we obtain

$$
\varphi_{x_{\imath} x_{\jmath}}+\frac{\phi_{x_{\imath}}}{\phi} \varphi_{x_{\jmath}}+\frac{\phi_{x_{\jmath}}}{\phi} \varphi_{x_{\imath}}=0,
$$

which is the equation (3.22). Similar way of equation (3.13), we get

$$
\begin{gathered}
\operatorname{Hess}_{\bar{g}} \varphi\left(Y_{\imath}, Y_{\jmath}\right)=Y_{\imath}\left(Y_{\jmath}(\varphi)\right)-\left({ }^{M} \nabla_{Y_{\imath}} Y_{\jmath}\right) \varphi \\
=\operatorname{Hess}_{F_{i}} \varphi_{k}\left(Y_{\imath}, Y_{\jmath}\right)+\frac{\sum_{i=1}^{m} b_{i}^{2} g_{F_{i}}\left(Y_{\imath}, Y_{\jmath}\right)}{b_{i}} \operatorname{grad}_{\bar{g}}\left(b_{i}\right)(\varphi) \\
=\frac{\sum_{i=1}^{m} b_{i}^{2} g_{F_{i}}\left(Y_{\imath}, Y_{\jmath}\right)}{b_{i}} \phi^{2} \sum_{k} \epsilon_{k}\left(b_{i}\right)_{x_{k}} \phi_{x_{k}}
\end{gathered}
$$

Then substituting equations (3.31), (3.33) and (3.34) in equation (3.28), we find

$$
\begin{gathered}
\left\{(r-1)\left(2 \phi \sum_{k} \epsilon_{k} \phi_{x_{k} x_{k}}-r \sum_{k} \epsilon_{k} \phi_{x_{k}}^{2}\right)\right. \\
-2 \sum_{i=1}^{m} s_{i} \frac{\phi^{2} \sum_{k} \epsilon_{k}\left(b_{i}\right)_{x_{k} x_{k}}-(n-2) \phi \sum_{k} \epsilon_{k} \phi_{x_{k}}\left(b_{i}\right)_{x_{k}}}{b_{i}} \\
+\sum_{i=1}^{m} \frac{\lambda_{F_{i}}}{b_{i}^{2}}-\sum_{i=1}^{m} s_{i}\left(s_{i}-1\right) \frac{\left(\phi^{2} \sum_{k} \epsilon_{k}\left(b_{i}^{2}\right)_{x_{k}}\right)}{b_{i}^{2}} \\
\left.-\sum_{i=1}^{m} \sum_{i=1, l \neq i}^{m} s_{i} s_{l} \frac{\left(\phi^{2} \sum_{k} \epsilon_{k}\left(b_{i}\right)_{x_{k}}\left(b_{l}\right)_{x_{k}}\right)}{b_{i} b_{l}}-\rho\right\}=\frac{\phi^{2}}{b_{i}} \sum_{k} \epsilon_{k} \phi_{x_{k}}\left(b_{i}\right)_{x_{k}} .
\end{gathered}
$$

The converse is a straightforward computation. This completes the proof. 
In order to obtain solutions for equations in Theorem 3.2, we consider $b_{i}, \phi$ and $\varphi$ invariant under the action of an $(r-1)$-dimensional translation group, and $\xi=\sum_{k=1}^{r} \alpha_{k} x_{k}, \alpha_{k} \in \mathbb{R}$ be a basic invariant for the $(r-1)$ dimensional translation group, then we get

Theorem 3.3. Let $\left(\mathbb{R}^{r}, g_{\mathbb{R}}\right)$ be a pseudo-Euclidean space, $r \geq 3$ with coordinates, $X=\left(X_{1}, \ldots, X_{r}\right)$ and $g_{i j}=\delta_{i j} \epsilon_{i j}$, let $M=\left(\mathbb{R}^{r}, \widetilde{g}\right) \times_{b_{1}} F_{1} \times_{b_{2}} F_{2} \times \ldots \times_{b_{m}} F_{m}$, be a multiply warped product where $\widetilde{g}=\frac{1}{\phi^{2}} g_{\mathbb{R}}$ and $F_{i}$ semi-Riemannian scalar-constant manifolds with curvatures $\lambda_{F_{i}}, t=\sum_{i=1}^{m} s_{i} \geq 1, b_{i}, \phi, \varphi: \mathbb{R}^{r} \rightarrow \mathbb{R}$, smooth functions and $b_{i}>0$. Consider the function $b_{i}(\xi), \phi(\xi)$ and $\varphi(\xi)$, where $\xi=\sum_{k=1}^{r} \alpha_{k} x_{k}, \alpha_{k} \in \mathbb{R}$ and $\sum_{k=1}^{r} \epsilon_{k} \alpha_{k}^{2}=\epsilon_{k_{0}}$ or $\sum_{k=1}^{r} \epsilon_{k} \alpha_{k}^{2}=0$. Then, the multiply warped product $(M, \bar{g})$ is a gradient Yamabe soliton with potential function $\varphi$ if and only if the functions $b_{i}, \phi, \varphi$ satisfy

$$
\begin{gathered}
\varphi^{\prime \prime}+2 \frac{\phi^{\prime}}{\phi} \varphi^{\prime}=0, \\
\epsilon_{k_{0}}\left\{(r-1)\left(2 \phi \phi^{\prime \prime}-r\left(\phi^{\prime}\right)^{2}\right)-2 \sum_{i=1}^{m} s_{i} \frac{\left(\phi^{2}\left(b_{i}\right)^{\prime \prime}-(n-2) \phi \phi^{\prime}\left(b_{i}\right)^{\prime}\right)}{b_{i}}\right. \\
\left.-\sum_{i=1}^{m} s_{i}\left(s_{i}-1\right) \frac{\left(\phi^{2}\left(b_{i}^{\prime}\right)^{2}\right)}{b_{i}^{2}}-\sum_{i=1}^{m} \sum_{i=1, l \neq i}^{m} s_{i} s_{l} \frac{\left(\phi^{2}\left(b_{i}\right)^{\prime}\left(b_{l}\right)^{\prime}\right)}{b_{i} b_{l}}+\phi \phi^{\prime} \varphi^{\prime}\right\} \\
=\rho-\sum_{i=1}^{m} \frac{\lambda_{F_{i}}}{b_{i}^{2}}, \\
\epsilon_{k_{0}}\left\{(r-1)\left(2 \phi \phi^{\prime \prime}-r\left(\phi^{\prime}\right)^{2}\right)-2 \sum_{i=1}^{m} s_{i} \frac{\left(\phi^{2}\left(b_{i}\right)^{\prime \prime}-(n-2) \phi \phi^{\prime}\left(b_{i}\right)^{\prime}\right)}{b_{i}}\right. \\
\left.-\sum_{i=1}^{m} s_{i}\left(s_{i}-1\right) \frac{\left(\phi^{2}\left(b_{i}^{\prime}\right)^{2}\right)}{b_{i}^{2}}-\sum_{i=1}^{m} \sum_{i=1, l \neq i}^{m} s_{i} s_{l} \frac{\left(\phi^{2}\left(b_{i}\right)^{\prime}\left(b_{l}\right)^{\prime}\right)}{b_{i} b_{l}}-\frac{\left(\sum_{i=1}^{m} b_{i}^{2}\right)}{b_{i}} \phi^{2}\left(b_{i}^{\prime}\right) \varphi^{\prime}\right\} \\
=\rho-\sum_{i=1}^{m} \frac{\lambda_{F_{i}}}{b_{i}^{2}},
\end{gathered}
$$

where $\epsilon_{k_{0}}=\sum_{k=1}^{r} \epsilon_{k} \alpha_{k}^{2}$. Moreover,

$$
\left(\varphi^{\prime \prime}+2 \frac{\phi^{\prime}}{\phi} \varphi^{\prime}\right)=0
$$

and

$$
\rho-\sum_{i=1}^{m} \frac{\lambda_{F_{i}}}{b_{i}^{2}}=0
$$

where $\sum_{k=1}^{r} \epsilon_{k} \alpha_{k}^{2}=0$.

Proof. Assume that $b_{i}(\xi), \phi(\xi)$ and $\varphi(\xi)$ are function of $\xi$, where $\xi=\sum_{k} \alpha_{k} x_{k}, \alpha_{k} \in \mathbb{R}$ and $\sum_{k=1}^{r} \epsilon_{k} \alpha_{k}^{2}=\epsilon_{k_{0}}$ or $\sum_{k=1}^{r} \epsilon_{k} \alpha_{k}^{2}=0$, then we obtain

$$
\begin{gathered}
\phi_{x_{\imath}}=\phi^{\prime} \alpha_{\imath}, \quad \phi_{x_{\imath} x_{\jmath}}=\phi^{\prime \prime} \alpha_{\imath} \alpha_{\jmath}, \quad\left(b_{i}\right)_{x_{\imath}}=\left(b_{i}\right)^{\prime} \alpha_{\imath}, \\
\left(b_{i}\right)_{x_{\imath} x_{\jmath}}=\left(b_{i}\right)^{\prime \prime} \alpha_{\imath} \alpha_{\jmath}, \varphi_{x_{\imath}}=\varphi^{\prime} \alpha_{\imath}, \varphi_{x_{\imath} x_{\jmath}}=\varphi^{\prime \prime} \alpha_{\imath} \alpha_{\jmath} .
\end{gathered}
$$


Replacing the equations (3.38) and (3.39) in (3.22), we find

$$
\left(\varphi^{\prime \prime}+2 \frac{\phi^{\prime}}{\phi} \varphi^{\prime}\right) \alpha_{\imath} \alpha_{\jmath}=0, \quad \forall \imath \neq \jmath .
$$

If there exist $\imath, \jmath, \imath \neq \jmath$ such that $\alpha_{\imath} \alpha_{\jmath} \neq 0$, then we have from the equation (3.40)

$$
\varphi^{\prime \prime}+2 \frac{\phi^{\prime}}{\phi} \varphi^{\prime}=0 .
$$

Similarly, using the equations (3.38) and (3.39) in the equations (3.23) and (3.24), we obtain

$$
\begin{gathered}
\left\{(r-1)\left(2 \phi \phi^{\prime \prime} \sum_{k} \epsilon_{k} \alpha_{k}^{2}-r\left(\phi^{\prime}\right)^{2} \sum_{k} \epsilon_{k} \alpha_{k}^{2}\right)\right. \\
-2 \sum_{i=1}^{m} s_{i} \frac{\left(\phi^{2}\left(b_{i}\right)^{\prime \prime} \sum_{k} \epsilon_{k} \alpha_{k}^{2}-(n-2) \phi \phi^{\prime}\left(b_{i}\right)^{\prime} \sum_{k} \epsilon_{k} \alpha_{k}^{2}\right)}{b_{i}} \\
+\sum_{i=1}^{m} \frac{\lambda_{F_{i}}}{b_{i}^{2}}-\sum_{i=1}^{m} s_{i}\left(s_{i}-1\right) \frac{\left(\phi^{2}\left(b_{i}^{\prime}\right)^{2} \sum_{k} \epsilon_{k} \alpha_{k}^{2}\right)}{b_{i}^{2}} \\
\left.-\sum_{i=1}^{m} \sum_{i=1, l \neq i}^{m} s_{i} s_{l} \frac{\left(\phi^{2}\left(b_{i}\right)^{\prime}\left(b_{l}\right)^{\prime} \sum_{k} \epsilon_{k} \alpha_{k}^{2}\right)}{b_{i} b_{l}}-\rho\right\} \frac{\epsilon_{l}}{\phi^{2}} \\
=\varphi^{\prime \prime} \alpha_{\imath}^{2}+2 \frac{\phi^{\prime}}{\phi} \varphi^{\prime} \alpha_{\imath}^{2}-\epsilon_{l} \frac{\phi^{\prime}}{\phi} \varphi^{\prime} \sum_{k} \epsilon_{k} \alpha_{k}^{2}
\end{gathered}
$$

and

$$
\begin{gathered}
\left\{(r-1)\left(2 \phi \phi^{\prime \prime} \sum_{k} \epsilon_{k} \alpha_{k}^{2}-r\left(\phi^{\prime}\right)^{2} \sum_{k} \epsilon_{k} \alpha_{k}^{2}\right)\right. \\
-2 \sum_{i=1}^{m} s_{i} \frac{\left(\phi^{2}\left(b_{i}\right)^{\prime \prime} \sum_{k} \epsilon_{k} \alpha_{k}^{2}-(n-2) \phi \phi^{\prime}\left(b_{i}\right)^{\prime} \sum_{k} \epsilon_{k} \alpha_{k}^{2}\right)}{b_{i}} \\
+\sum_{i=1}^{m} \frac{\lambda_{F_{i}}}{b_{i}^{2}}-\sum_{i=1}^{m} s_{i}\left(s_{i}-1\right) \frac{\left(\phi^{2}\left(b_{i}^{\prime}\right)^{2} \sum_{k} \epsilon_{k} \alpha_{k}^{2}\right)}{b_{i}^{2}} \\
\left.-\sum_{i=1}^{m} \sum_{i=1, l \neq i}^{m} s_{i} s_{l} \frac{\left(\phi^{2}\left(b_{i}\right)^{\prime}\left(b_{l}\right)^{\prime} \sum_{k} \epsilon_{k} \alpha_{k}^{2}\right)}{b_{i} b_{l}}-\rho\right\} \\
=\frac{\left(\sum_{i=1}^{m} b_{i}^{2}\right)}{b_{i}} \phi^{2}\left(b_{i}^{\prime}\right) \varphi^{\prime} \sum_{k} \epsilon_{k} \alpha_{k}^{2} .
\end{gathered}
$$

By the use of the equation (3.41) and $\sum_{k} \epsilon_{k} \alpha_{k}^{2}=\epsilon_{k_{0}}$ in (3.42), we find

$$
\epsilon_{k_{0}}\left\{(r-1)\left(2 \phi \phi^{\prime \prime}-r\left(\phi^{\prime}\right)^{2}\right)-2 \sum_{i=1}^{m} s_{i} \frac{\left(\phi^{2}\left(b_{i}\right)^{\prime \prime}-(n-2) \phi \phi^{\prime}\left(b_{i}\right)^{\prime}\right)}{b_{i}}\right.
$$




$$
\left.-\sum_{i=1}^{m} s_{i}\left(s_{i}-1\right) \frac{\left(\phi^{2}\left(b_{i}^{\prime}\right)^{2}\right)}{b_{i}^{2}}-\sum_{i=1}^{m} \sum_{i=1, l \neq i}^{m} s_{i} s_{l} \frac{\left(\phi^{2}\left(b_{i}\right)^{\prime}\left(b_{l}\right)^{\prime}\right)}{b_{i} b_{l}}+\phi \phi^{\prime} \varphi^{\prime}\right\}=\rho-\sum_{i=1}^{m} \frac{\lambda_{F_{i}}}{b_{i}^{2}} .
$$

In the same way, using equation (3.41) and $\sum_{k} \epsilon_{k} \alpha_{k}^{2}=\epsilon_{k_{0}}$ in (3.43), we get

$$
\begin{gathered}
\epsilon_{k_{0}}\left\{(r-1)\left(2 \phi \phi^{\prime \prime}-r\left(\phi^{\prime}\right)^{2}\right)-2 \sum_{i=1}^{m} s_{i} \frac{\left(\phi^{2}\left(b_{i}\right)^{\prime \prime}-(n-2) \phi \phi^{\prime}\left(b_{i}\right)^{\prime}\right)}{b_{i}}\right. \\
-\sum_{i=1}^{m} s_{i}\left(s_{i}-1\right) \frac{\left(\phi^{2}\left(b_{i}^{\prime}\right)^{2}\right)}{b_{i}^{2}}-\sum_{i=1}^{m} \sum_{i=1, l \neq i}^{m} s_{i} s_{l} \frac{\left(\phi^{2}\left(b_{i}\right)^{\prime}\left(b_{l}\right)^{\prime}\right)}{b_{i} b_{l}} \\
\left.-\frac{\left(\sum_{i=1}^{m} b_{i}^{2}\right)}{b_{i}} \phi^{2}\left(b_{i}^{\prime}\right) \varphi^{\prime}\right\}=\rho-\sum_{i=1}^{m} \frac{\lambda_{F_{i}}}{b_{i}^{2}} .
\end{gathered}
$$

Hence, if we take $\sum_{k} \epsilon_{k} \alpha_{k}^{2}=\epsilon_{k_{0}}$, then we obtain the equations (3.35), (3.36) and (3.37).

Now, taking $\sum_{k} \epsilon_{k} \alpha_{k}^{2}=0$ in equations (3.35), (3.36) and (3.37), we have

$$
\left(\varphi^{\prime \prime}+2 \frac{\phi^{\prime}}{\phi} \varphi^{\prime}\right)=0
$$

and

$$
\rho-\sum_{i=1}^{m} \frac{\lambda_{F_{i}}}{b_{i}^{2}}=0 .
$$

Now, we need to consider the case $\alpha_{k_{0}}=1$ and $\alpha_{k}=0$ for $k \neq k_{0}$. In this case, equation (3.40) is trivially satisfied and since equation (3.43) does not depend on the index $\imath$, we have that equation (3.43) is equivalent to equation (3.37). Finally, we need to show the validity of equation (3.35) and (3.36). Taking $\imath=k_{0}$, that is, $\alpha_{k_{0}}=1$, in (3.42), we obtain

$$
\begin{gathered}
\left\{(r-1)\left(2 \phi \phi^{\prime \prime} \epsilon_{k_{0}}-r\left(\phi^{\prime}\right)^{2} \epsilon_{k_{0}}\right)-2 \sum_{i=1}^{m} s_{i} \frac{\left(\phi^{2}\left(b_{i}\right)^{\prime \prime} \epsilon_{k_{0}}-(n-2) \phi \phi^{\prime}\left(b_{i}\right)^{\prime} \epsilon_{k_{0}}\right)}{b_{i}}\right. \\
\left.+\sum_{i=1}^{m} \frac{\lambda_{F_{i}}}{b_{i}^{2}}-\sum_{i=1}^{m} s_{i}\left(s_{i}-1\right) \frac{\left(\phi^{2}\left(b_{i}^{\prime}\right)^{2} \epsilon_{k_{0}}\right)}{b_{i}^{2}}-\sum_{i=1}^{m} \sum_{i=1, l \neq i}^{m} s_{i} s_{l} \frac{\left(\phi^{2}\left(b_{i}\right)^{\prime}\left(b_{l}\right)^{\prime} \epsilon_{k_{0}}\right)}{b_{i} b_{l}}-\rho\right\} \frac{\epsilon_{k_{0}}}{\phi^{2}} \\
=\varphi^{\prime \prime}+\frac{\phi^{\prime}}{\phi} \varphi^{\prime}
\end{gathered}
$$

and for $\imath=k_{0}$, that is, $\alpha_{\imath}=0$, we get

$$
\begin{gathered}
\left\{(r-1)\left(2 \phi \phi^{\prime \prime} \epsilon_{k_{0}}-r\left(\phi^{\prime}\right)^{2} \epsilon_{k_{0}}\right)-2 \sum_{i=1}^{m} s_{i} \frac{\left(\phi^{2}\left(b_{i}\right)^{\prime \prime} \epsilon_{k_{0}}-(n-2) \phi \phi^{\prime}\left(b_{i}\right)^{\prime} \epsilon_{k_{0}}\right)}{b_{i}}\right. \\
\left.+\sum_{i=1}^{m} \frac{\lambda_{F_{i}}}{b_{i}^{2}}-\sum_{i=1}^{m} s_{i}\left(s_{i}-1\right) \frac{\left(\phi^{2}\left(b_{i}^{\prime}\right)^{2} \epsilon_{k_{0}}\right)}{b_{i}^{2}}-\sum_{i=1}^{m} \sum_{i=1, l \neq i}^{m} s_{i} s_{l} \frac{\left(\phi^{2}\left(b_{i}\right)^{\prime}\left(b_{l}\right)^{\prime} \epsilon_{k_{0}}\right)}{b_{i} b_{l}}-\rho\right\} \frac{\epsilon_{l}}{\phi^{2}} \\
=-\epsilon_{\imath} \epsilon_{k_{0}} \frac{\phi^{\prime}}{\phi} \varphi^{\prime} .
\end{gathered}
$$

However, the last equation are equivalent to equations (3.35) and (3.36). This completes the proof. 


\section{References}

[1] Barbosa, E. and Ribeiro, E., On conformal solutions of the yamabe flow. Archiv der Mathematik 101 (2013), 79-89.

[2] Cao, H-D., Sun, X. and Zhang, Y., On the structure of gradient yamabe solitons. Mathematical Research Letters 19 (2012), 767-774.

[3] De, U. C., Turan, M., Yıldız, A. and De, A., Ricci solitons and gradient Ricci solitons on 3-dimensional normal almost contact metric manifolds. Publicationes Mathematica Debrecen 80 (2012), 127-142.

[4] Dobarro, F. and Ünal, B., Curvature of multiply warped products. Journal of Geometry and Physics 55 (2005), 75-106.

[5] Feitosa, F. E. S., Freitas, A. A. and Gomes, J. N. V., On the construction of gradient Ricci soliton warped product. Nonlinear Analysis 161 (2017), 30-43.

[6] He, C., Petersen, P. and Wylie, W., Warped product rigidity. Asian J. Math. 19 (2015), 135-170.

[7] He, C., Gradient Yamabe solitons on warped products. arXiv preprint arXiv:1109.2343, (2011).

[8] Karaca, F. and Özgür, C., Gradient Ricci Solitons on Multiply Warped Product Manifolds. Filomat 32 (2018), 4221-4228.

[9] Lee, S. D., Kim, B. H. and Choi, J. H., On a classification of warped product spaces with gradient Ricci solitons. The Korean Journal of Mathematics 24 (2016), 627-636.

[10] Lee, S. D., Kim, B. H. and Choi, J. H., Warped product spaces with Ricci conditions. Turkish Journal of Mathematics 41 (2017), $1365-1375$.

[11] Ma, L. and Miquel, V., Remarks on scalar curvature of Yamabe solitons. Annals of Global Analysis and Geometry 42 (2012), 195-205.

[12] Neto, B. L. and Tenenblat, K., On gradient yamabe solitons conformal to a pseudoeuclidian space. Journal of Geometry and Physics 123 (2018), 284-291.

[13] Sousa, M. L. and Pina, R., Gradient ricci solitons with structure of warped product. Results in Mathematics 71(3-4) (2017), 825-840.

[14] Ünal, B., Doubly warped products. Ph.D. Thesis, University of Missouri-Columbia, 2000.

[15] Ünal, B., Multiply warped products. Journal of Geometry and Physics 34 (2000), 287-301.

[16] Tokura, W., Adriano, L., Pina, R. and Barboza, M., On warped product gradient Yamabe solitons. Journal of Mathematical Analysis and Applications 473(1) (2019), 201-214.

[17] Turan, M., De, U. C. and Yıldız, A., Ricci solitons and gradient Ricci solitons in three-dimensional trans-Sasakian manifolds. Filomat 26(2) (2012), 363-370

\section{Affiliations}

FATMA KARACA

ADDRESs: Beykent University, Department of Mathematics, 34550, Istanbul-Turkey.

E-MAIL: fatmagurlerr@gmail.com

ORCID ID: : 0000-0002-0382-8028 